\title{
Nurses' Expectations of an Inpatient Portal for Hospitalized Patients and Caregivers
}

\author{
Peter L. T. Hoonakker ${ }^{1}$ Rebecca J. Rankin ${ }^{2}$ Jennifer C. Passini ${ }^{3}$ Jenny A. Bunton ${ }^{4}$ \\ Bradley D. Ehlenfeldt ${ }^{4}$ Shannon M. Dean ${ }^{3}$ Anne S. Thurber ${ }^{3}$ Michelle M. Kelly ${ }^{3}$
}

${ }^{1}$ Center for Quality and Productivity Improvement, University of Wisconsin-Madison, Madison, Wisconsin, United States

2 Department of Nursing, Nursing Informatics, University of Wisconsin Hospitals and Clinics, Madison, Wisconsin, United States

${ }^{3}$ Department of Medicine, University of Wisconsin Hospitals and Clinics, Madison, Wisconsin, United States

${ }^{4}$ Department of Health Information Services, University of Wisconsin

Hospitals and Clinics, Madison, Wisconsin, United States

\author{
Address for correspondence Peter L. T. Hoonakker, PhD, Center for \\ Quality and Productivity Improvement (CQPI), University of \\ Wisconsin-Madison, 3124 Engineering Centers Building, 1550 \\ Engineering Drive, Madison, WI 53706, United States \\ (e-mail: peter.hoonakker@wisc.edu).
}

Appl Clin Inform 2019;10:625-633.

Abstract

Keywords

- patient portal

- inpatient

- implementation

- expectations

- nurses and staff personnel
Background Patient portals are intended to engage patients and enhance patientcentered care. Recent studies suggest that the information within portals could provide benefits to patients and their caregivers during hospitalization; however, few studies have examined nurse and staff expectations of portals when used in the hospital setting.

Objective This article examines inpatient nurse and support staff expectations of a commercially available inpatient portal prior to its hospital-wide implementation.

Methods In this cross-sectional study, nurses and support staff were surveyed 1 month prior to the implementation of an inpatient portal for patients. Items included respondent characteristics, satisfaction with online inpatient portal training, expectations of the effects of portal use on patients, caregivers, and staff, overall acceptance, and barriers to its implementation.

Results Of 881 respondents, $73.0 \%$ were staff nurses, $18.4 \%$ nurse assistants, $4.3 \%$ unit coordinators, and $1.2 \%$ nurse managers. Respondents were generally satisfied with the portal information they received from online training. A majority liked the portal to some extent prior to its use (66.7\%); however, they noted multiple implementation barriers, including: tablets would get lost/damaged (66.2\% of respondents), patients and/or caregivers would have too many questions (48.5\%), and staff would have problems integrating it into their workflow (44.7\%). Respondents working on medical units had higher expectations $(p<0.001)$ and acceptance $(p<0.01)$ of the portal than those on surgical and intensive care units. Nurse managers were more positive than respondents with other job roles were (all $p<0.001$ ).

Conclusion Overall, nurse and support staff had high expectations of the effects of inpatient portal use prior to its hospital-wide implementation. They thought it would benefit patients and/or their caregivers; however, they also perceived several barriers to its implementation. These results will be used in conjunction with patient and caregiver perspectives to inform future efforts to evaluate and improve upon inpatient portal implementation and dissemination across health systems. received

March 19, 2019

accepted after revision June 29, 2019 (c) 2019 Georg Thieme Verlag KG Stuttgart . New York
DOI https://doi.org/

10.1055/s-0039-1694750. ISSN 1869-0327. 


\section{Background and Significance}

Patients who are better informed about their health are more engaged in their care, which can result in improved outcomes. ${ }^{1-3}$ Patient portals have been introduced as a tool to facilitate clinicians' communication of information with patients. These portals are intended to promote engagement by providing patients with timely, online access to their electronic health record (EHR) information and by supporting communication through secure messaging with their clinicians. $^{4,5}$ Patient portals have primarily been used to share information with patients in the ambulatory setting. ${ }^{5-8}$

The use of patient portals in the inpatient setting is a more recent phenomenon. These "inpatient portals" provide hospitalized patients with access to information from their inpatient EHR and a way to recognize and communicate with their inpatient clinicians. ${ }^{9}$ Published literature has focused primarily on inpatient portal design ${ }^{10-13}$ and use on single units with specific patient populations (e.g., surgical patients). ${ }^{14}$ Patients and caregivers participants have generally shown willingness to use these portals and found them useful. $9,15-19$

Less is known about clinician expectations of inpatient portals and the effects of use on their work, particularly among inpatient nurses and support staff. ${ }^{20}$ Pell et $\mathrm{al}^{21}$ evaluated hospitalized patients' (50) and clinicians' (28 physicians; 14 nurses) experiences using an inpatient portal before and after implementation on a single medical unit. Most thought the portal would improve care quality, but also increase their workload. Kelly et $\mathrm{al}^{22}$ examined the effects of inpatient portal use on clinicians working on a pediatric medical/surgical unit before and 6 months after implementation and found similar results. Only one published study has evaluated health care team perceptions of an inpatient portal after system-wide implementation across multiple hospitals. ${ }^{23}$ Physicians (193), nurses (439), and support staff (186) were generally positive about the portal 1 month after implementation and thought that patients would use and benefit from it. Whether clinicians working on different hospital units have different expectations of the effects of inpatient portal use is unknown.

\section{Objective}

The objective of this study was to examine nurse and support staff expectations of a commercially available inpatient portal (MyChart Bedside, Epic Systems) prior to its hospital-wide implementation. What are their expectations of the effects of inpatient portal use on patients, caregivers, and staff? What are the barriers to implementation? Are there differences in expectations across units (i.e., medical, surgical, or intensive care) and job roles (i.e., staff nurse, nurse assistant, unit coordinator, or nurse manager)? Results from this study will be used to evaluate whether nurse and support staff perceptions change over time and to support future efforts to improve inpatient portal design, implementation, and dissemination.

\section{Methods}

\section{Study Design, Setting, and Participants}

In this cross-sectional study, nurses and support staff were surveyed 1 month prior to the implementation of an inpatient portal for patients hospitalized at a 592-bed, quaternary care, Midwest teaching hospital. At this organization, a system-wide EHR (Epic Systems, Verona, Wisconsin, United States) was implemented in 2008 and an ambulatory patient portal (MyChart, Epic Systems) in 2009. The inpatient portal (MyChart Bedside, Epic Systems) was first pilot-tested on a unit within the adjoining children's hospital in 2015. ${ }^{17,22}$

Prior to inpatient portal use across the entire adult hospital, nurses and support staff were required to complete an online training. At the end of the training, they were asked to fill out a voluntary survey about their expectations of the inpatient portal. Eligible participants included health unit coordinators (HUCs), nursing assistants (NAs), registered nurses, clinical nurse specialists (CNSs), and nurse managers who worked primarily on a medical, surgical, or intensive care unit (ICU).

\section{Inpatient Portal and Implementation Process}

MyChart Bedside ( - Fig. 1) is an inpatient, EHR application on a tablet computer. It allows inpatients and/or their caregivers to access information specific to their hospital stay, such as their vital signs, medication list and administration times, laboratory results, daily schedule, pictures of their health care team members, and a way to send preset requests. Vital signs, problem lists, and medications are released in real time. Laboratory results are released every 30 minutes from 7:00 a.m. to 11:30 p.m. Other test results, such as pathology reports, culture, and radiology results, are not released to the portal. Tablets can only be used within the hospital. They included other applications such as games, music, and video streaming; but did not include access to the Internet. For a more detailed description of the inpatient portal, see Kelly et al ${ }^{17}$ and Walker et al. ${ }^{24}$ Over 1 year, a multidisciplinary implementation team met monthly to plan inpatient portal implementation. This 31-member team included a project manager and representatives from stakeholder groups, including: bedside nursing (2), nurse management (2), support staff (2), physicians, trainees, patient/family advisors, inpatient informatics, learning and development, patient experience, quality and safety, hospital administration, ambulatory patient portal, security, and legal. They made decisions regarding tablet storage, provisioning and security, functionalities offered, laboratory releases, institution-specific education, response times, and staff online training development. The hospital expectations for staff were described as follows: Patients received the tablet from the HUC as part of the admission process, or, when the HUC was not available, from the Care Team Leader. The nurse caring for the patient was responsible for explaining the purpose and functionalities of the portal and respond to questions. All requests made through the portal were directed to an EHR work list that was monitored by the HUC. The HUC was expected to respond in-person to requests or through the portal within 15 to 30 minutes and/or ask for assistance 


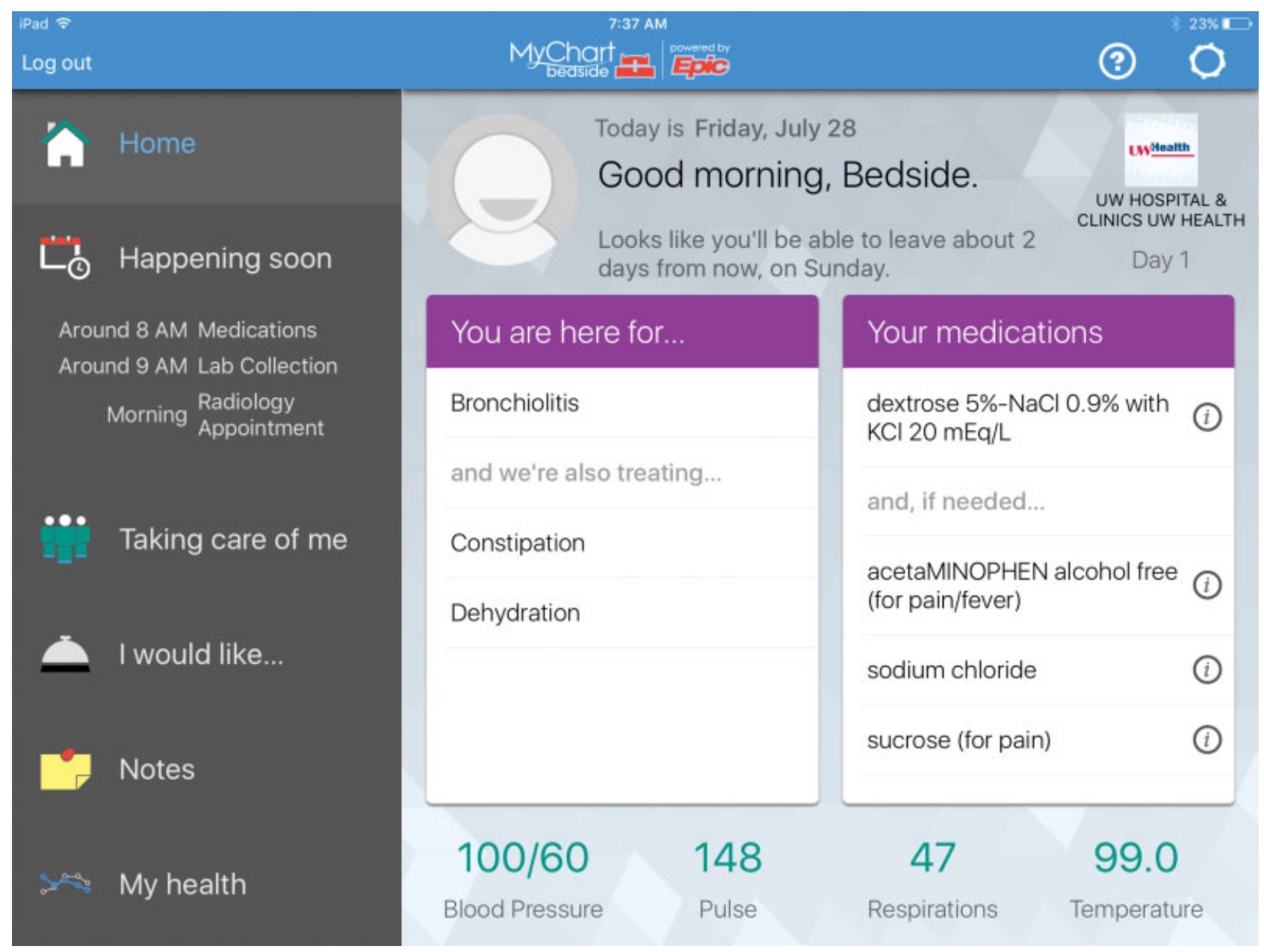

Fig. 1 Inpatient portal home screen (MyChart Bedside, screenshot, 2019 Epic Systems Corporation, used with permission).

from the patient's nurse or physician. More information about the inpatient portal and its implementation can be found in this free, online toolkit: https://www.hipxchange. org/InpatientPortal.

\section{Data Collection Procedure}

In August 2017, staff were sent an invitation to complete the required online training module. This training consisted of an overview of the rationale for using the inpatient portal, how to issue a tablet, a description of portal features, hospital expectations for staff, and who to call with technical issues. The online training also included feedback from patients, families, and staff obtained during the pilot study in our children's hospital. ${ }^{17}$ At the completion of the training, staff were prompted with a link to complete a voluntary, online survey. Survey responses were anonymous. The study was performed in compliance with the World Medical Association Declaration of Helsinki on Ethical Principles for Medical Research Involving Human Subjects, and was reviewed by the University of Wisconsin-Madison Institutional Review Board.

\section{Survey}

We constructed an online Qualtrics survey using items from the published literature ${ }^{25-28}$ that were adapted after pilot- ing and review by the implementation team. This 32-item survey consisted of items assessing: (1) respondent characteristics (job role, unit, age, education, general technology use), (2) satisfaction with information received in the online training $^{28}$ (sufficient - insufficient, timely - not timely, useful - useless, on a 9-point scale), (3) expectations of the effects of inpatient portal use on patients, caregivers, and staff ${ }^{25-28}$ (e.g., patient-nurse/doctor communication, respondent workload, satisfaction, care quality, and error reduction on a 5-point Likert scale, 1-strongly disagree, 3neither agree nor disagree, and 5-strongly agree), (4) overall acceptance of the inpatient portal ${ }^{28}$ (1-Dislike very much, don't want to use to 10 -Like very much, eager to use), and (5) barriers to implementation. Questions on barriers were adapted from previous literature 22,27 and consisted of predetermined items and an "other" free-text option. The entire survey can be found at: https:/cqpi. wiscweb.wisc.edu/wp-content/uploads/sites/599/2018/07/ MCB-Pre-Implementation-Survey.pdf .

\section{Data Analysis}

Survey data were downloaded into SPSS (Version 20). Staff who self-identified as working on units in which the inpatient portal would not be used (e.g., postanesthesia care, psychiatric, research, and prisoner units) or those working 
across multiple units (e.g., float nurses) were excluded from data analysis. Due to the small number of CNS and nurse manager respondents, these job roles were combined into one category ("nurse management").

Survey items on satisfaction with information received in the online training were recoded and combined to create a satisfaction with online training scale (1-Very bad, 9-Very good). Survey items on expectations of the impact of inpatient portal use (after recoding of the only negatively worded item on workload) were combined into an expectations of the inpatient portal scale (1-Very bad, 5-Very good). The responses to the 5-point Likert response categories on the expectations items were also combined into three categories: (1) disagree (strongly disagree and disagree), (2) neither agree nor disagree, and (3) agree (strongly agree and agree) to create -Fig. 2. A variable was created to count how many barriers to implementation each respondent perceived. Cronbach's $\alpha$ of the satisfaction with online training scale was 0.90 and of the expectations of the inpatient portal scale was 0.93. To assess for differences between groups, we used the chisquare test for nominal data and the nonparametric MannWhitney and Kruskal-Wallis tests for ordinal data. We used the $t$-test and one-way analysis of variance to compare scale scores.

\section{Results}

In all, 1,304 staff completed the online training and 1,265 filled out the survey (response rate 97\%). Data from those who did not identify as working primarily on a medical, surgical, or ICU where the inpatient portal would be used were excluded $(N=261$, e.g., float nurses or staff who worked in postanesthesia care, psychiatric, research, prisoner, or combined units). Data from those that had more than $50 \%$ of their data missing $(N=123)$ were also excluded. Thus, responses from 881 staff were included in the analysis.

Respondent characteristics are shown in -Table 1. A majority were staff nurses $(73.0 \%), \leq 44$ years old $(83.0 \%)$, and reported using technology on a daily basis (91.6\%).

\section{Satisfaction with the Online Training}

Respondents were generally satisfied with the information they received about the inpatient portal from online training. Most found the information sufficient (85.2\% reported a score lower than the midpoint 5; 1-sufficient, 9-insufficient; mean $=2.54$ [standard deviation (SD) 1.66]), useful (82.9\% reported a score lower than 5; 1-useful, 9-useless; mean $=2.71$ [SD 1.75]), and timely (77.9\% reported a score lower than 5; 1-timely, 9-not timely; mean $=2.98$ [SD 2.02]).

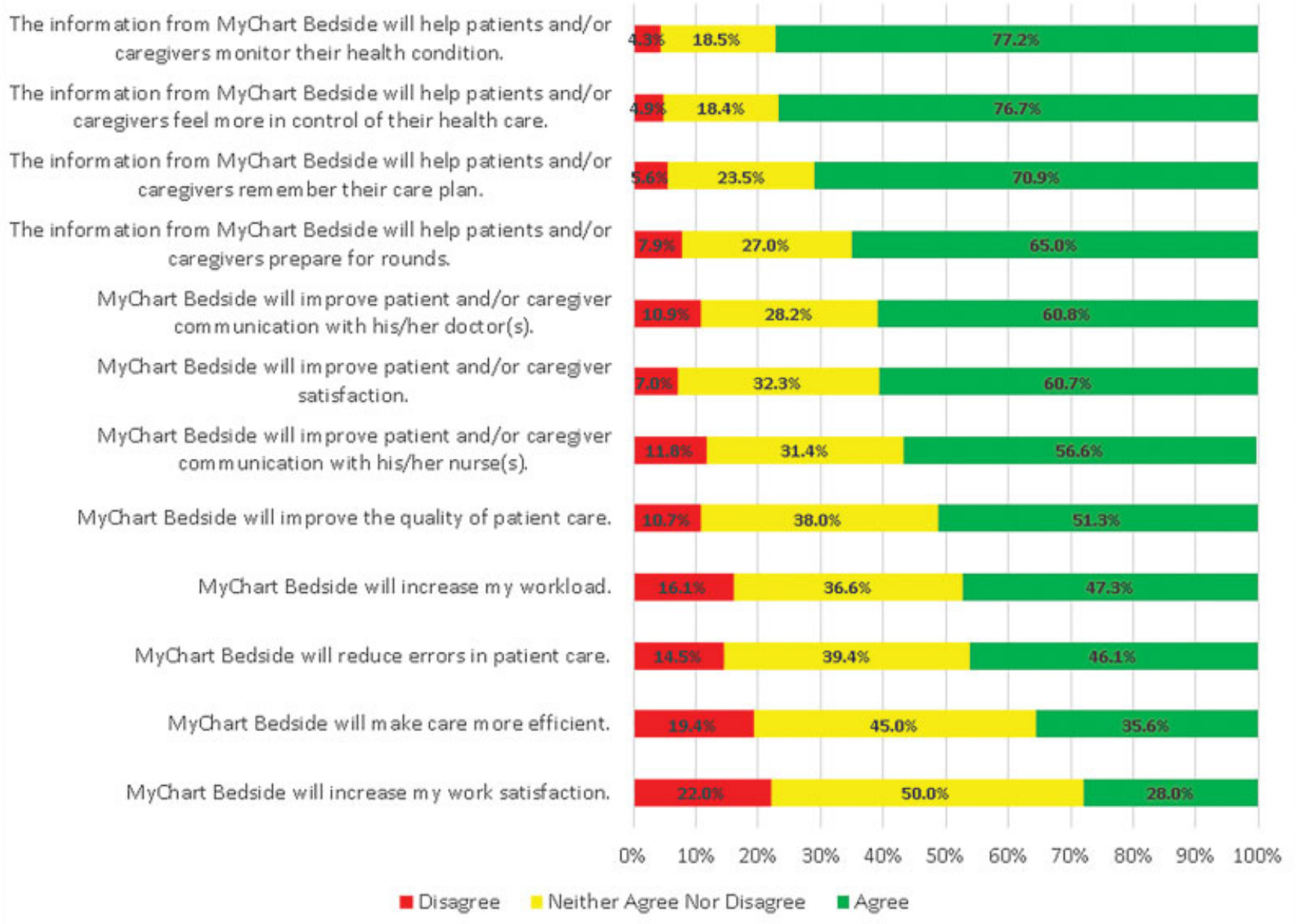

Fig. 2 Respondents' expectations of the impact of the inpatient portal (MyChart Bedside, Epic Systems) on patients, caregivers, and staff. 
Table 1 Nurse and support staff respondent characteristics $(N=881)$

\begin{tabular}{|l|l|l|}
\hline Respondent characteristics & $N$ & Percent \\
\hline Job title & & \\
\hline Health unit coordinator & 38 & 4.3 \\
\hline Nurse assistant & 162 & 18.4 \\
\hline Staff nurse & 643 & 73.0 \\
\hline Nurse management & 11 & 1.2 \\
\hline Other/Missing & 27 & 3.1 \\
\hline Unit & & \\
\hline Medical & 359 & 40.7 \\
\hline Surgical & 245 & 27.8 \\
\hline Intensive care & 277 & 31.4 \\
\hline Age & & \\
\hline 18-24 & 193 & 21.9 \\
\hline 25-34 & 380 & 43.1 \\
\hline 35-44 & 159 & 18.0 \\
\hline 45-54 & 88 & 10.0 \\
\hline 55 or older & 36 & 4.1 \\
\hline Missing & 25 & 2.8 \\
\hline Highest level of education & & \\
\hline Some college or 2-year degree & 155 & 17.6 \\
\hline 4-year college graduate & 555 & 63.0 \\
\hline More than 4-year college degree & 129 & 14.6 \\
\hline High school or GED & 20 & 2.3 \\
\hline Missing & 22 & 2.5 \\
\hline Technology use & 42 & 4.8 \\
\hline Daily & 807 & 0.9 \\
\hline Several times a week & & \\
\hline Once a week or less & & \\
\hline Missing & & \\
\hline
\end{tabular}

Abbreviation: GED, General Equivalency Diploma.

\section{Expectations about of the Effects of Inpatient Portal Use on Patients, Caregivers, and Staff}

Most respondents expected that inpatient portal use would provide benefits for patients and/or their caregivers (-Fig. 2). For example, $77.2 \%$ of respondents agreed that the information from the inpatient portal would help patients and/or caregivers monitor their health condition. Respondents were more neutral about how the inpatient portal would affect their own work. For example, 52.7\% felt that the inpatient portal would not increase their workload or were unsure.

\section{Overall Acceptance and Barriers to Implementation of the Inpatient Portal}

A majority of respondents liked the inpatient portal to some extent before implementation (66.7\% reported a score higher than 5 ; 1-Dislike very much, 10 -Like very much; mean $=6.57$
[SD 2.42]). Respondent perceptions of the barriers to implementation are summarized in - Fig. 3. Respondents had considerable concerns, including: tablets would get lost or damaged (66.2\% of respondents), patients and/or caregivers would have too many questions about the information in the inpatient portal (48.5\%), staff would have problems integrating it into their workflow (44.7\%), and staff would be skeptical of it (37.0\%).

\section{Differences in Responses across Units and Job Roles}

Overall, respondents working on medical units had higher expectations of the inpatient portal and acceptance than those on surgical and ICUs ( - Table 2, $p<0.001$ and $<0.01$, respectively).

Results in -Table 3 show that nurse management was more positive about the inpatient portal than other respondents were (all $p<0.001$ ). These managers had higher expectations about the potential effects of inpatient portal use. They also had higher overall acceptance and anticipated fewer barriers to implementation of the inpatient portal when compared with respondents with other job roles.

\section{Discussion}

Although the use of inpatient portals to engage patients in the hospital setting is growing, relatively little is known about clinician perceptions of their use and implementation. This is the first large study examining nurse and support staff perceptions of an inpatient portal before hospital-wide implementation. Results suggest respondents were satisfied with the training they received, with the majority finding the online module providing sufficient, timely, and useful information. Many nurses and support staff had high expectations of how inpatient portal use could potentially benefit patients and caregivers; however, they were more neutral about how use would affect their own work. Nearly half expected that it would increase their workload and a third thought it would be an additional frustration to their workday. Respondents working on medical units had higher expectations and acceptance than those on surgical and ICUs and nurse management was more positive than HUCs and bedside nurses. Results of this study can be used to facilitate inpatient portal implementation, by addressing the barriers perceived by staff.

Our results are similar to those found in other studies evaluating clinician expectations prior to inpatient portal implementation on single units. Before its implementation hospital-wide, we piloted the use of this inpatient portal on a single unit of a children' hospital at the same health care organization. ${ }^{22}$ Clinicians' expectations that inpatient portal use would improve communication, care quality, and patient safety were also high, but only partially realized 6 months after implementation. Clinicians cited similar barrier to implementation (e.g., tablets would get lost or damaged); however, both these barriers and their pervasive expectations about how use would negatively affect their workflow and workload did not materialize. We 
The tablets will get lost or damaged

Patients and/or caregivers will have too many questions about the information from Mychart Bedside

I will be too busy to incorporate it into my workflow

Staff will be skeptical of it

There will not be enough computer technical support

It will be an added frustration to my workday

Patients and/or car egivers who use it will know test results before the patient's doctor or nurse

The information will not be useful for patients and/or caregivers

It will be too hard for me to learn to use

Other
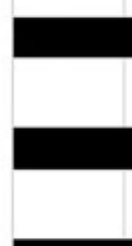

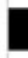

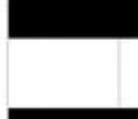

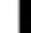

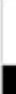

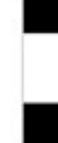
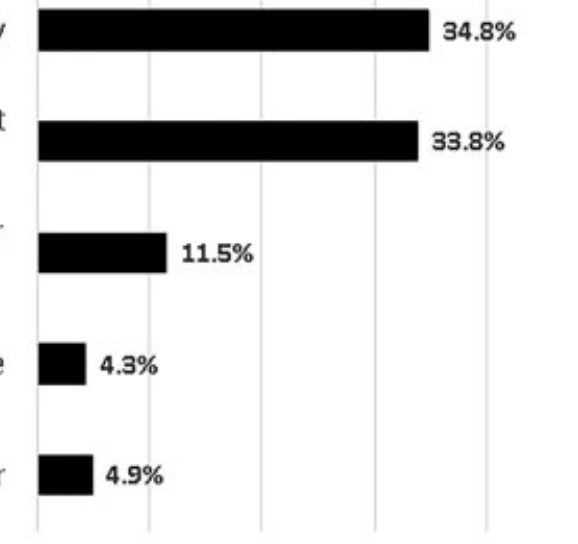

Table 2 Difference in respondent satisfaction with online training, expectations, acceptance, and barriers to implementation of the inpatient portal by unit

\begin{tabular}{|c|c|c|c|c|c|}
\hline & $\begin{array}{l}\text { All } \\
(N=881)\end{array}$ & Medical $(N=359)$ & Surgical $(N=245)$ & $\begin{array}{l}\text { Intensive care } \\
(N=277)\end{array}$ & p-Value \\
\hline & \multicolumn{4}{|c|}{ Mean [standard deviation] } & \\
\hline $\begin{array}{l}\text { Satisfaction with online training } \\
\text { (scaled from } 1 \text {-very bad, 10-very good) }\end{array}$ & $\begin{array}{l}7.26 \\
{[1.67]}\end{array}$ & $\begin{array}{l}7.37 \\
{[1.60]}\end{array}$ & $\begin{array}{l}7.15 \\
{[1.73]}\end{array}$ & $\begin{array}{l}7.22 \\
{[1.69]}\end{array}$ & 0.23 \\
\hline $\begin{array}{l}\text { Expectations of the inpatient portal } \\
\text { (scaled from 1-very bad, 5-very good) }\end{array}$ & $\begin{array}{l}3.38 \\
{[0.54]}\end{array}$ & $\begin{array}{l}3.47 \\
{[0.54]}\end{array}$ & $\begin{array}{l}3.33 \\
{[0.51]}\end{array}$ & $\begin{array}{l}3.31 \\
{[0.56]}\end{array}$ & $<0.001$ \\
\hline $\begin{array}{l}\text { Acceptance of the inpatient portal } \\
\text { (1-dislike very much, } 10 \text {-like very much) }\end{array}$ & $\begin{array}{l}6.57 \\
{[2.42]}\end{array}$ & $\begin{array}{l}6.90 \\
{[2.39]}\end{array}$ & $\begin{array}{l}6.40 \\
{[2.38]}\end{array}$ & $\begin{array}{l}6.29 \\
{[2.46]}\end{array}$ & $<0.01$ \\
\hline Barriers to implementation (count) & $\begin{array}{l}3.22 \\
{[1.94]}\end{array}$ & $\begin{array}{l}3.11 \\
{[1.86]}\end{array}$ & $\begin{array}{l}3.41 \\
{[2.04]}\end{array}$ & $\begin{array}{l}3.19 \\
{[1.95]}\end{array}$ & 0.17 \\
\hline
\end{tabular}


Table 3 Difference in respondent satisfaction with online training, expectations, acceptance, and barriers to implementation of the inpatient portal by role

\begin{tabular}{|c|c|c|c|c|c|c|}
\hline & $\begin{array}{l}\text { All } \\
(N=854)\end{array}$ & $\begin{array}{l}\text { Health unit } \\
\text { coordinator } \\
(N=38)\end{array}$ & $\begin{array}{l}\text { Nurse } \\
\text { assistant } \\
(N=162)\end{array}$ & $\begin{array}{l}\text { Staff nurse } \\
(N=643)\end{array}$ & $\begin{array}{l}\text { Nurse } \\
\text { management } \\
(N=11)\end{array}$ & $p$-Value \\
\hline & \multicolumn{5}{|c|}{ Mean [standard deviation] } & \\
\hline $\begin{array}{l}\text { Satisfaction with online training } \\
\text { (scaled from 1-very bad, } 10 \text {-very good) }\end{array}$ & $\begin{array}{l}7.27 \\
{[1.63]}\end{array}$ & $\begin{array}{l}6.99 \\
{[1.87]}\end{array}$ & $\begin{array}{l}7.73 \\
{[1.46]}\end{array}$ & $7.16[1.65]$ & $\begin{array}{l}7.91 \\
{[1.28]}\end{array}$ & $<0.001$ \\
\hline $\begin{array}{l}\text { Expectations of the inpatient portal } \\
\text { (scaled from 1-very bad, 5-very good) }\end{array}$ & $\begin{array}{l}3.38 \\
{[0.53]}\end{array}$ & $\begin{array}{l}3.52 \\
{[0.52]}\end{array}$ & $\begin{array}{l}3.50 \\
{[.62]}\end{array}$ & $3.33[0.51]$ & $\begin{array}{l}3.70 \\
{[0.40]}\end{array}$ & $<0.001$ \\
\hline $\begin{array}{l}\text { Acceptance of the inpatient portal } \\
\text { (1-dislike very much, 10-like very much) }\end{array}$ & $\begin{array}{l}6.54 \\
{[2.43]}\end{array}$ & $\begin{array}{l}7.47 \\
{[1.90]}\end{array}$ & $\begin{array}{l}7.22 \\
{[2.43]}\end{array}$ & $6.27[2.40]$ & $\begin{array}{l}9.36 \\
{[0.92]}\end{array}$ & $<0.001$ \\
\hline Barriers to implementation (count) & $\begin{array}{l}3.26 \\
{[1.94]}\end{array}$ & $\begin{array}{l}2.21 \\
{[1.45]}\end{array}$ & $\begin{array}{l}2.91 \\
{[1.91]}\end{array}$ & $3.44[1.94]$ & $\begin{array}{l}1.73 \\
{[1.10]}\end{array}$ & $<0.001$ \\
\hline
\end{tabular}

units and ICUs. Further, the online training included feedback from non-ICU families and staff from our pilot study at our children's hospital. Additional research is needed to examine inpatient portal use by different patient populations and if and why inpatient portal use might affect personnel on the units differently. Hospitals should consider even broader stakeholder representation on teams tasked with inpatient portal implementation, specifically staff on surgical units and ICUs. Training may also be tailored to better meet the needs of staff on units caring for different patient populations. .

Nurse management was more positive than NAs, HUCs, and in particular staff nurses about inpatient portal implementation. This may be explained by multiple factors. Nurse managers have been in higher-level meetings, tend to have more buy-in to organizational changes, and, thus, usually have heard messages from the top for longer than front-line staff. Nurses are also most exposed to potential consequences of inpatient portal implementation, such as patients or their caregiver(s) asking questions about the information on the portal. Future work should consider including physicians and other ancillary staff who may have unique perspectives about inpatient portal implementation.

Several limitations should be considered. In this crosssectional study, data were collected from nurses and support staff before use of a commercially available inpatient portal (MyChart Bedside, Epic Systems) in one academic hospital in the Midwest, which may limit its generalizability. Nurses were not "exposed" to an inpatient portal in their practice (although they may have experiences with the outpatient portal), which may make it difficult to know what to expect. The online training may also have had a positive or negative impact on their perceptions and expectations. Further, although the survey we used was based on earlier work with implementation of the inpatient portal in a children's hospital, responses were limited to the range of expectations and perceived barriers by the questions that were provided in survey questions. There may be other perceptions or barriers that are important, but were not assessed in this study. The six nurses and support staff who were on the implementa- tion team could have been survey participants; however, the total number of survey participants exceeded 880 . Thus, any potential impact their responses may have had on results is likely minimal. Future research should elicit the perspectives of patients, caregivers, physicians, and other ancillary staff.

Overall, nursing and support staff had high expectations of the use of an inpatient portal to engage hospitalized patients and their caregivers; however, they also perceived several barriers to its implementation. Some expected that the patient portal would increase their workload and that it would be an added frustration to their workday. When implementing the inpatient portal, the implementation team and management should address these concerns. After inpatient portal implementation, it should be checked whether these concerns have actually materialized, and if inpatient portal implementation does result in an increase in workload and frustration, those issues should be addressed.

\section{Clinical Relevance Statement}

Patient portals have the potential to engage parents of hospitalized children and may play a role in improving the safety and quality of inpatient care. ${ }^{7-9}$ Several studies have shown that, although physicians may have some concerns about the inpatient portal implementation, ${ }^{21,22}$ generally patients are very satisfied. ${ }^{9}$ However, few studies have examined nurses' expectations about inpatient portal implementation. In this study, we examined what barriers and benefits, nurses and nursing staff expected prior to inpatient implementation. Nurses and nursing staff saw several potential benefits, but also barriers. By addressing these barriers, inpatient portal implementation could be improved.

\section{Multiple Choice Questions}

1. Hospitals are rapidly implementing inpatient portals. Patients (and/or their caregivers) are provided with a tablet that contains medical information, such as a 
problem list, medications, and laboratory results. Several studies have shown that, despite some concerns from physicians, in general, patients and their caregivers are very satisfied with the information provided. However, in very few studies hospital nurses and nursing staff have been asked what they think about inpatient portal implementation. What was the most frequent barrier to implementation reported by nurses and nursing staff in this study?

a. It would increase nurses' and nursing staff workload.

b. Patients would have too many questions.

c. The information would not be useful to patients.

d. The tablets would get lost or stolen.

Correct Answer: The correct answer is option d. Nearly two-thirds of nurses and nursing staff were afraid that the tablets provided by the hospital would get lost or stolen. They were also concerned about the other issues mentioned.

2. Nurses and nursing staff also anticipated benefits to inpatient portal implementation. What did they most frequently report as a potential benefit?

a. Patients (and their caregivers) would have fewer questions.

b. The inpatient portal would reduce errors.

c. The inpatient portal would increase patient satisfaction.

d. The information on the inpatient portal would help patients and/or caregivers monitor their health condition.

Correct Answer: The correct answer is option d. Nurses and nursing staff saw several potential benefits to inpatient portal implementation, but the most respondents reported that the information would help patients and/or their caregivers. They thought the portal would help patients (and/or their caregivers): (1) monitor their health condition, (2) feel more in control of their care, and (3) remember their care plan.

\section{Protection of Human and Animal Subjects}

This study was performed in compliance with the World Medical Association Declaration of Helsinki on Ethical Principles for Medical Research Involving Human Subjects, and, as quality improvement, was deemed exempt from full review by the University of Wisconsin-Madison Institutional Review Board.

\section{Funding}

This work was supported by the Department of Pediatrics Research and Development Grant at the University of Wisconsin School of Medicine and Public Health. This publication was also supported by the Clinical and Translational Science Award program through the National Center for Advancing Translational Sciences, grant UL1TR000427. Funding sources had no involvement in study design, analysis, or interpretation of data.

\section{Conflict of Interest}

None declared.
Acknowledgments

We would like to thank nursing and support staff and the portal implementation and steering committees for their valuable feedback and support.

\section{References}

1 Hibbard JH, Greene J. What the evidence shows about patient activation: better health outcomes and care experiences; fewer data on costs. Health Aff (Millwood) 2013;32(02):207-214

2 Dalal AK, Dykes PC, Collins S, et al. A web-based, patient-centered toolkit to engage patients and caregivers in the acute care setting: a preliminary evaluation. J Am Med Inform Assoc 2016;23(01): 80-87

3 Sawesi S, Rashrash M, Phalakornkule K, Carpenter JS, Jones JF. The impact of information technology on patient engagement and health behavior change: a systematic review of the literature. JMIR Med Inform 2016;4(01):e1

4 Tang PC, Ash JS, Bates DW, Overhage JM, Sands DZ. Personal health records: definitions, benefits, and strategies for overcoming barriers to adoption. J Am Med Inform Assoc 2006;13(02): 121-126

5 Powell KR. Patient-perceived facilitators of and barriers to electronic portal use: a systematic review. Comput Inform Nurs 2017; 35(11):606-607

6 Haun JN, Lind JD, Shimada SL, et al. Evaluating user experiences of the secure messaging tool on the Veterans Affairs' patient portal system. J Med Internet Res 2014;16(03):e75

7 Irizarry T, DeVito Dabbs A, Curran CR. Patient portals and patient engagement: a state of the science review. J Med Internet Res 2015;17(06):e148

8 Mook PJ, Trickey AW, Krakowski KE, et al. Exploration of portal activation by patients in a healthcare system. Comput Inform Nurs 2018;36(01):18-26

9 Kelly MM, Coller RJ, Hoonakker PLT. Inpatient portals for hospitalized patients and caregivers: a systematic review. J Hosp Med 2018;13(06):405-412

10 Vawdrey DK, Wilcox LG, Collins SA, et al. A tablet computer application for patients to participate in their hospital care. AMIA Annu Symp Proc 2011;2011:1428-1435

11 Caligtan CA, Carroll DL, Hurley AC, Gersh-Zaremski R, Dykes PC. Bedside information technology to support patient-centered care. Int J Med Inform 2012;81(07):442-451

12 Wilcox L, et al. Designing inpatient technology to meet the medication information needs of cardiology patients. In Proceedings of the 2nd ACM SIGHIT International Health Informatics Symposium. ACM: Miami, FL, USA; 2012:831-836

13 Dykes PC, Carroll DL, Hurley AC, et al. Building and testing a patient-centric electronic bedside communication center. J Gerontol Nurs 2013;39(01):15-19

14 Robinson JR, Davis SE, Cronin RM, Jackson GP. Use of a patient portal during hospital admissions to surgical services. AMIA Annu Symp Proc 2017;2016:1967-1976

15 Patmon FL, Gee PM, Rylee TL, Readdy NL. Using interactive patient engagement technology in clinical practice: a qualitative assessment of nurses' perceptions. J Med Internet Res 2016;18 (11):e298

16 Woollen J, Prey J, Wilcox L, et al. Patient experiences using an inpatient personal health record. Appl Clin Inform 2016;7(02): 446-460

17 Kelly MM, Hoonakker PLT, Dean SM. Using an inpatient portal to engage families in pediatric hospital care. J Am Med Inform Assoc 2017;24(01):153-161

18 Lee J, Kim JGB, Jin M, et al. Beneficial effects of two types of personal health record services connected with electronic medical records within the hospital setting. Comput Inform Nurs 2017; 35(11):574-581 
19 McAlearney AS, Fareed N, Gaughan A, MacEwan SR, Volney J, Sieck CJ. Empowering patients during hospitalization: perspectives on inpatient portal use. Appl Clin Inform 2019;10(01):103-112

20 Tiase VL. ANI Emerging Leaders Project: nurses' perceptions of the use of health information technology tools for patient and family engagement. Comput Inform Nurs 2015;33(12):520-522

21 Pell JM, Mancuso M, Limon S, Oman K, Lin CT. Patient access to electronic health records during hospitalization. JAMA Intern Med 2015;175(05):856-858

22 Kelly MM, Dean SM, Carayon P, Wetterneck TB, Hoonakker PL. Healthcare team perceptions of a portal for parents of hospitalized children before and after implementation. Appl Clin Inform 2017;8(01):265-278

23 Hefner JL, Sieck CJ, Walker DM, Huerta TR, McAlearney AS. System-wide inpatient portal implementation: survey of health care team perceptions. JMIR Med Inform 2017;5(03):e31
24 Walker DM, Menser T, Yen PY, McAlearney AS. Optimizing the user experience: identifying opportunities to improve use of an inpatient portal. Appl Clin Inform 2018;9(01):105-113

25 Hoonakker PL, Cartmill RS, Carayon P, Walker JM. Development and psychometric qualities of the SEIPS survey to evaluate CPOE/ EHR implementation in ICUs. Int J Healthc Inf Syst Inform 2011;6 (01):51-69

26 Cox ED, Carayon P, Hansen KW, et al. Parent perceptions of children's hospital safety climate. BMJ Qual Saf 2013;22(08):664-671

27 Leveille SG, Walker J, Ralston JD, Ross SE, Elmore JG, Delbanco T. Evaluating the impact of patients' online access to doctors' visit notes: designing and executing the OpenNotes project. BMC Med Inform Decis Mak 2012;12:32

28 Carayon P, Cartmill R, Blosky MA, et al. ICU nurses' acceptance of electronic health records. J Am Med Inform Assoc 2011;18(06): 812-819 